\title{
Whole grain intake and nutrient health in Irish adults
}

\author{
N. F. C. Devlin ${ }^{1}$, B. A. McNulty ${ }^{1}$, J. Walton ${ }^{2}$, A. Flynn ${ }^{2}$, M. J. Gibney ${ }^{1}$ and A. P. Nugent ${ }^{1}$ \\ ${ }^{1}$ UCD Institute of Food and Health, University College Dublin, Belfield, Dublin 4, Ireland and ${ }^{2}$ School of Food and \\ Nutritional Sciences, University College Cork, Cork, Ireland
}

Regular consumption of wholegrain foods has been promoted to improve health and reduce the risk of developing certain diseases. The aim of this study was to quantify wholegrain intakes in a representative sample of Irish adults using the National Adult Nutrition Survey (NANS; ages 18-90 years, n1051 after the exclusion of under-reporters) ${ }^{1}$, (www.iuna.net) and to compare nutrient intakes in consumers versus non-consumers of whole grain. A 4 day food diary was used to examine habitual food and beverage consumption in this survey to include brand information and ingredient listings. In the current analysis, the presence or absence of wholegrain was confirmed at food and brand level using food package labelling, comparable products and a recipe database specific to NANS.

\begin{tabular}{|c|c|c|c|c|c|c|}
\hline & \multicolumn{5}{|c|}{ Tertiles of mean daily whole grain intakes } & \multirow[b]{2}{*}{ Corrected $P$} \\
\hline & Non-consumers $n=94$ & Tertile $1 n=298$ & Tertile $2 n=313$ & Tertile $3 n=346$ & $P$ & \\
\hline Energy (MJ) & $9 \cdot 0^{\mathrm{a}}$ & $9 \cdot 4^{\mathrm{ab}}$ & $9 \cdot 1^{\mathrm{a}}$ & $9.7^{\mathrm{b}}$ & $<0.001$ & ** \\
\hline$\%$ energy from protein & $15 \cdot 6^{\mathrm{a}}$ & $15 \cdot 8^{\mathrm{a}}$ & $16.7^{\mathrm{b}}$ & $16.9^{\mathrm{b}}$ & $<0.001$ & ${ }^{*} *$ \\
\hline$\%$ energy from carbohydrate & $40 \cdot 4^{\mathrm{a}}$ & $41 \cdot 6^{\mathrm{ab}}$ & $42 \cdot 5^{\mathrm{b}}$ & $44 \cdot 6^{\mathrm{c}}$ & $<0.001$ & ** \\
\hline$\%$ energy from total sugar & $15 \cdot 5^{\mathrm{a}}$ & $16 \cdot 9^{\mathrm{ab}}$ & $16 \cdot 8^{\mathrm{ab}}$ & $17 \cdot 8^{\mathrm{b}}$ & 0.002 & $*$ \\
\hline$\%$ energy from fat & $36 \cdot 3^{\mathrm{a}}$ & $35 \cdot 1^{\mathrm{ab}}$ & $33.9^{\mathrm{bc}}$ & $33 \cdot 2^{\mathrm{c}}$ & $<0.001$ & ** \\
\hline$\%$ energy from saturated fat & $14 \cdot 1^{\mathrm{a}}$ & $13.9^{\mathrm{a}}$ & $13 \cdot 4^{\mathrm{ab}}$ & $12 \cdot 9^{\mathrm{b}}$ & 0.001 & $*$ \\
\hline Fibre/energy (g/10MJ) & $17 \cdot 0^{\mathrm{a}}$ & $19 \cdot 3^{\mathrm{b}}$ & $23 \cdot 1^{\mathrm{c}}$ & $26 \cdot 8^{\mathrm{d}}$ & $<0.001$ & ${ }^{*} *$ \\
\hline Sodium/energy (mg/10MJ) & 2892.9 & $2958 \cdot 9$ & $2949 \cdot 0$ & 2869.9 & NS & NS \\
\hline Iron/energy (mg/10MJ) & $16 \cdot 4$ & 17.0 & 17.5 & $18 \cdot 2$ & NS & NS \\
\hline Calcium/energy (mg/10MJ) & $1088 \cdot 6^{\mathrm{ab}}$ & $1059 \cdot 3^{\mathrm{a}}$ & $1114 \cdot 4^{\mathrm{a}}$ & $1206 \cdot 6^{\mathrm{b}}$ & $<0.001$ & \\
\hline Potassium/energy (mg/10MJ) & $3518 \cdot 8^{\mathrm{ab}}$ & $3351 \cdot 8^{\mathrm{a}}$ & $3647 \cdot 1^{\mathrm{bc}}$ & $3802 \cdot 7^{\mathrm{c}}$ & $<0.001$ & ** \\
\hline Phosphorus/energy (mg/10MJ) & $1452 \cdot 2^{\mathrm{a}}$ & $1508 \cdot 5^{\mathrm{a}}$ & $1657.6^{\mathrm{b}}$ & $1737 \cdot 9^{\mathrm{c}}$ & $<0.001$ & ${ }^{* *}$ \\
\hline Magnesium/energy (mg/10MJ) & $299 \cdot 2^{\mathrm{a}}$ & $311 \cdot 0^{\mathrm{a}}$ & $349 \cdot 3^{\mathrm{b}}$ & $382.7^{\mathrm{c}}$ & $<0.001$ & ** \\
\hline Thiamin/energy (mg/10MJ) & $2 \cdot 2$ & $3 \cdot 2$ & 3.8 & 3.9 & NS & NS \\
\hline Riboflavin/energy (mg/10MJ) & $2 \cdot 5$ & 3.2 & $4 \cdot 2$ & $4 \cdot 1$ & NS & NS \\
\hline Niacin/energy (mg/10MJ) & $32 \cdot 1$ & $30 \cdot 3$ & $35 \cdot 2$ & 33.6 & NS & NS \\
\hline Vitamin B6/energy (mg/10MJ) & 3.5 & $4 \cdot 3$ & 4.9 & $5 \cdot 3$ & NS & NS \\
\hline Vitamin D/energy $(\mu \mathrm{g} / 10 \mathrm{MJ})$ & $4 \cdot 3^{\mathrm{a}}$ & $5 \cdot 0^{\mathrm{a}}$ & $5 \cdot 3^{\mathrm{ab}}$ & $6 \cdot 6^{\mathrm{b}}$ & 0.008 & NS \\
\hline
\end{tabular}

abc Values with unlike superscript letters denote significant differences between the mean values of whole grain intake across the tertiles of whole grain intake (General

Linear Model adjusted for gender); NS: not significant; Corrected $P$ values refer to Bonferroni correction at a family level ${ }^{*} P<0 \cdot 0027$; ${ }^{* *} P<0 \cdot 001$

Mean intakes (g/day) of wholegrain were 38.4 for Irish adults, rising to 42.2 in consumers only $(91 \cdot 1 \%)$. Wholegrain consumers had more nutrient dense diets for a number of micronutrients, with lower intakes of fat and saturated fat, and higher intakes of protein and carbohydrate (\% total energy). Future work will explore the consumption patterns of whole grain foods and investigate associations with markers of nutritional status and metabolic health.

This analysis was funded by the Department of Agriculture, Food and the Marine and the Health Research Board under Nutridata Project Ref $13 \mathrm{~F} 542$.

1. IUNA (2011) National Adult Nutrition Survey Summary Report. Available from: http://www.iuna.net/wp-content/uploads/2010/12/ National-Adult-Nutrition-Survey-Summary-Report-March-2011.pdf 